\title{
LUCARELLI Carlo. - Le temps des hyènes
}

\section{Alain Gascon}

\section{OpenEdition}

\section{Journals}

\section{Édition électronique}

URL : https://journals.openedition.org/etudesafricaines/30617

DOI : 10.4000/etudesafricaines.30617

ISSN : 1777-5353

\section{Éditeur}

Éditions de l'EHESS

\section{Édition imprimée}

Date de publication : 15 juin 2020

Pagination : 450-454

ISBN : 978-2-7132-2828-5

ISSN : 0008-0055

\section{Référence électronique}

Alain Gascon, " LuCARELLI Carlo. - Le temps des hyènes », Cahiers d'études africaines [En ligne], 238| 2020, mis en ligne le 15 juin 2020, consulté le 03 janvier 2023. URL : http://journals.openedition.org/ etudesafricaines/30617 ; DOI : https://doi.org/10.4000/etudesafricaines.30617

Ce document a été généré automatiquement le 3 janvier 2023

Tous droits réservés 


\title{
LUCARELLI Carlo. - Le temps des hyènes
}

\author{
Alain Gascon
}

\section{RÉFÉRENCE}

LUCARELl Carlo. - Le temps des hyènes . Paris, Métailié, 2018, 192 p. Traduit de l'italien par Serge Quadruppani (Il tempo delle iene, Turin, Einaudi, 2015).

1 Avant d'aborder le roman de Carlo Lucarelli dans les Cahiers, je tiens à répondre aux interrogations que suscite la recension d'un ouvrage de fiction dont l'auteur est italien, donc citoyen d'un État qui colonisa l'Érythrée pendant un demi-siècle. Rappelons que des écrivains, même européens, ont prononcé de violents réquisitoires contre le système colonial et Lucarelli est de ceux-ci. Mais, au début de son roman, il remercie son épouse érythréenne, descendante d'Ogbà, son héros « indigène ». On sait aussi que l'opinion publique est plus directement touchée par l'émotion qui se dégage des œuvres de fiction que par les faits rapportés et analysés par des publications académiques ou des rapports administratifs. En outre, le roman policier, longtemps considéré comme un genre mineur, sort d'Europe ou des États-Unis et explore le monde tout en abordant maintenant des sujets « sérieux ».

2 La publication, en France, du grand roman historique, à prétexte policier, la $8^{e}$ vibration, 1 , a révélé Carlo Lucarelli, un écrivain ambitieux quant à son style, son récit et son sujet. Il est très connu en Italie, comme auteur de romans policiers et de scénarios. Dans cette fresque romanesque en forme d'opéra, il peint la bataille d'Adwa (1896), défaite italienne majeure, avec les images d'Apocalypse Now de Coppola et les accents du Götterdämmerung («Crépuscule des Dieux») de Wagner. En outre, preuves à l'appui, il soutient que les Italiens, tout juste unifiés par la Maison de Savoie, n'étaient guère différents des Éthiopiens, alors rassemblés sous la rude férule de Menilek. Angelo Turco (Université de l'Aquila) et moi avons porté nos "regards croisés » et avons passé au crible ce grand roman ${ }^{2}$. Dans le livre suivant Albergo Italia ${ }^{3}$, la courte intrigue policière semble prendre le pas, alors qu'elle entraîne le lecteur dans l'après-Adwa, dans les recoins les plus sombres de la société érythréenne où coloniaux et colonisés se côtoient 
et s'observent. Le titre de l'ouvrage illustre l'indécision des autorités qui hésitaient sur le sort de la Colonia Eritrea: colonie d'implantation ou hôtel pour des séjours temporaires ${ }^{4}$. Rappelons que Mussolini, officiellement "Fondatore dell'Impèro Italiano" en 1936, avait organisé en 1911-1912, alors socialiste, des manifestations insurrectionnelles à Ancône d'où partait le corps expéditionnaire vers la Lybie. Enfin, notons combien les traductions remarquables de Serge Quadruppani ont contribué au sujet des romans de Carlo Lucarelli.

Dans Le temps des hyènes, le capitaine des carabiniers royaux Piero Colaprico, secondé par son adjoint (zaptié) Ogbagabriel Ogbà, le «Sherlock Holmes abyssin », reprend du service car trois indigènes et un marquis pendent aux branches d'un grand sycomore. Comme dans Albergo Italia, Lucarelli perd le lecteur dans une intrigue policière à tiroirs, au cours de laquelle il brosse le portrait-charge des coloniaux. On retrouve l'atmosphère poisseuse de Conrad, de Simenon ou de Céline, mais aussi le propos du roman inachevé de Camus, Le premier homme. En Algérie comme en Érythrée, les premiers colons étaient aussi misérables que les "indigènes" auxquels ils étaient censés apporter les bienfaits de la civilisation. L'avenir de la colonie, après le désastre d'Adwa, n'est pas encore assuré, mais le gouverneur Martini (1897-1907) s'efforce de préserver les restes de l'empire colonial italien avorté. Interrogé par le capitaine, Colombi répond: "[...] sans même savoir si on allait rester en Afrique » (p.50) et Colaprico demande au procureur: "Si nous restons en Afrique» (p.132). Comme bien des colonies, l'Érythrée attira un échantillon représentatif des irréguliers et des inadaptés accourus de toute la péninsule, de toute l'Europe et même des États-Unis.

Outre son style aérien et son humour qui captivent son lecteur, Lucarelli fait preuve d'un grand souci de vraisemblance par l'usage des dialectes italiens et du tigrinia. Le traducteur français réussit la gageure de rendre compte de la diversité des dialectes parlés par les Italiens qui se comprennent à peine alors que, sur les hautes terres en deçà et au-delà de la frontière, les "Abyssins " parlent tigrigna, langue transcrite et traduite par l'auteur. En outre, il fait référence au contexte historique, mais surtout, il enracine son récit dans les territoires qui participent à l'action. Afalba/Afelba, où furent découverts les pendus, apparaît dans les cartes de la Guida de $1929^{5}$ (pp. 608-609) et de $1938^{6}$ (pp. 192-193), à mi-chemin entre Decameré et Saganeiti, sur les plateaux au sud d'Asmära. L'édition de 1929 (p. 656) précise : Afalba (2237 m.) «Il paese è a sin. su rocca dominato da una chiesa cattolica». L'édition de 1938 (p. 287) ajoute : «tipico villaggio eritrei » [...] « lasciando a d. la chiesa copta (sic.)»; " Nel 1894 il villaggio, per essersi rifiutato di aderire alla rivolta contro gl'Italiani ${ }^{7}$, fu duram. trattato dai ribelli » et à $7 \mathrm{~km}$ : « il sicomoro di Maaraba, all'ombra del quale si tennero memorabile dispute tra eritrei cattolici e copti ». Même si Colaprico le déplore, les chaleurs ont desservi Massaoua-Bașé, qui a perdu son rang de capitale de la colonie, pour la fraîcheur d'Asmära et des hauteurs où l'auteur nous promène à dos de mulet ou en calèche. Ainsi, quand il cherche Taklè Maryam près d'Asmära, l'auteur lui fait dire in petto: "Cela rappela à Colaprico [originaire de Toscane] les villages de Sicile» (p. 139).

5 Lucarelli a bâti son récit suivant le principe de la symétrie entre coloniaux et colonisés, pour encore accentuer leurs ressemblances, alors qu'ils vivent dans un monde en principe divisé. Seuls les carabiniers, - même si Colaprico affirme : "c'est connu, les nègres n'ont aucune envie de travailler » (p. 27) -, les ascari (les supplétifs indigènes), les «madames » (les concubines indigènes des Italiens, p. 189) et Oualla, la prostituée, en somme des marginaux, s'affranchissent des interdits, au cœur même de la société 
coloniale. Taklè Maryam, l'épouse abäša (abyssine) du marquis, fille de däğazmač (noble militaire), rencontre le brigadier, mais en territoire " neutre ", l'Albergo Italia, avant de retourner avec sa fille métisse au Tegray, en Éthiopie. L'auteur fait le parallèle entre la vengeance traditionnelle et sanglante de Bashà Busrù, le chef de village et le meurtre, selon le rituel des anarchistes romagnols, de l'abominable Ciacci commis par le carabinier Ravagli, également auteur d'exécutions extra-judiciaires d'Érythréens, soupçonnés de sympathie pour Menilek, dans les dunes proches de Massaoua (p. 108). La vie n'avait pas grand prix dans la colonie: trois Érythréens pendus seraient vite oubliés s'il n'y avait un marquis italien suspendu au même grand sycomore. Ce livre rappelle, après bien d'autres, combien l'éloignement érode les principes moraux dans les colonies où l'on « africanise » Hobbes : «l'homme [y] est une hyène pour l'homme. » Sur les rives de la mer Rouge, sommeillait en chaque Européen, un trafiquant et un meurtrier, un Monfreid en somme, qui ne demandait qu'à s'éveiller. Lucarelli insiste aussi, à juste titre, sur les scandales financiers qui accompagnèrent les conquêtes coloniales au point qu'ils en furent inséparables: trafic de monnaies, compagnies fantômes, mines imaginaires, plantations qui n'existaient que sur le papier et concessions marginales et menacées comme la terre octroyée à la mère de Marguerite Duras qui subissait les assauts de l'océan ${ }^{8}$. Dans le livre de Lucarelli, la veuve italienne éplorée du marquis, amour de jeunesse de Colaprico, s'associe à un notaire véreux, en vue de provoquer un coup de bourse, en répandant le bruit de la découverte d'or dans une fausse mine, d'en vendre des actions et ensuite de disparaître. Je reviendrai plus loin sur l'or érythréen dont il a été récemment question.

Lucarelli traite des aspects les plus sombres de la colonisation italienne qu'Angelo Dell Bocca $^{9}$ a dénoncés dans un livre qui a fait, et qui fait toujours, polémique en Italie. Témoignages et documents irréfutables à l'appui, ce dernier a démonté le mythe d'une colonisation italienne différente, de "braves gens », plutôt paternalistes. Dans ses trois romans consacrés à l'Érythrée, il conforte son réquisitoire. Le temps des hyènes, plus que les livres précédents, pose la question de la naissance du séparatisme et du nationalisme en Érythrée qui fut loin d'être un sentiment partagé par tous les Érythréens, même au temps de la guerre d'indépendance (1958-1991) ${ }^{10}$. Le frère d'Ogbà, Woldeab, agriculteur frappé comme lui par la grave crise climatique des années 1890, s'engage comme lui, mais dans l'armée du negus Menilek avec un fusil Vetterli dérobé aux Italiens (p. 95). On nous précise aussi que la région frontalière est remplie d'ascari déserteurs de l'armée italienne qui cherchent à passer au Tegray comme Taklé Maryam qui, elle, quitte la colonie où sa fille métisse, Lèm Lèm, n'aura aucun avenir. Ainsi, des Érythréens, pourtant éduqués par les Italiens, ayant constaté qu'ils ne pourraient jamais sortir de leur condition inférieure, ont franchi le cours du Maräb pour se mettre au service de l'Éthiopie ${ }^{11}$. Abba Jérôme et surtout Lorenzo Täezaz occupaient ainsi des postes importants auprès du negus lors du conflit de 1936. Avant de rallier tardivement les indépendantistes du Front populaire de libération d'Érythrée (FPLPE), Tadlä Bairu servit Haylä Sellasé. Le général Aman Mikaél Andom, originaire d'Érythrée et éphémère chef de l'État éthiopien en 1974 après la chute du monarque, avait participé à la résistance contre les Italiens qui avaient pourtant réussi à mobiliser la moitié de la main-d'œuvre de la colonie dans l'invasion de l'Éthiopie, selon Irma Taddia ${ }^{12}$.

7 On appréciera également la recette de la préparation du repas fait de crêpes d'enğära et de wätṭ (ragoût) par Manna, la femme d'Ogbà, recueillie par sa petite-fille, épouse de Carlo Lucarelli. Toutefois, ne dit-on pas plutôt zegeni en tigrinia? Les éthiopisantsérythréïsants, et aussi les autres, liront avec profit Le temps des hyènes qui poursuit et 
renouvelle, sur un mode mineur, la grande symphonie de la $8^{e}$ Vibration. Ce livre, à la fois distrayant, sérieux et grave, prend une dimension actuelle avec l'ouverture - définitive espérons-le - des frontières de l'Érythrée et avec la paix avec l'Éthiopie, mais, comme le rappelle Ogbà : « Il n'y a rien de plus trompeur que l'évidence » (p. 190).

En guise de conclusion, signalons que la presse, il y a un an ou deux, a rapporté qu'une entreprise canadienne avait obtenu la concession de mines d'or sur les hautes terres en Érythrée. En effet, il y a de l'or car, au Tegray au sud de l'Érythrée, l'érosion a dégagé des laves du tertiaire, les couches sédimentaires secondaires et primaires et sculpté les fameux amba calcaires. Puis, au nord du Maräb, émerge, en Érythrée, le socle antécambrien qui renferme les filons de métaux précieux. L'extraction de l'or a certes fait progresser le PNB érythréen, mais c'est l'entourage du président et le président luimême qui dirigent l'entreprise d'État chapotant l'ensemble des entreprises du pays, et qui en ont tiré les bénéfices. Donc, toutes les mines d'or ne sont pas toutes factices en Érythrée. Des ONG canadiennes ont attaqué, devant leur Cour suprême, l'entreprise qui n'a exigé aucune garantie quant aux conditions de travail dans les mines, que l'on sait déplorables.

\section{NOTES}

1. C. LuCARELLI, $8^{e}$ vibration, Paris, Métailié, 2010.

2. A. GASCON \& A. TuRCo, « Regards croisés sur la $8^{\mathrm{e}}$ Vibration de Carlo Lucarelli », Pount, 6, 2012, pp. 187-199.

3. C. LuCARELLI, Albergo Italia, Paris, Métailié, 2016.

4. A. Gascon, « Compte-rendu d'Albergo Italia », Pount, 12, 2018, pp. 205-209.

5. Guida d'Italia del TCI, Possedimenti e Colonie, Milan, Touring Club Italiano, 1929.

6. Guida d'Italia della Consociazione Turistica Italiana, Africa Orientale Italiana, Milan, Consociazione Turistica Italiana, 1938.

7. Il s'agit de la révolte dirigée par Bahta Hagos contre les projets de colonisation agricole des hautes terres.

8. Voir M. DURAS, Un barrage contre le Pacifique, Paris, Gallimard, 1950.

9. G. MEYNIER, « Mémoires italiennes de la colonisation. À propos de : Italiani, brava gente? d'Angelo Del Boca », Esprit, janvier 2008, pp. 36-51.

10. B. TAFLA, «Eritrea in Retrospect. An Excerpt from the Memoirs of Fitawrari Mika'el Hasama Rakka », Africa (Roma), Anno LX (1), Marzo 2005, pp. 1-64.

11. L'interprète du gouverneur Martini, Gäbrä-Egziabehér, renseignait les Éthiopiens. Voir A. ROUAUD, Deux intellectuels éthiopiens face à l'Italie Gäbrä-Egziabehér Gilay et Afä wärq Gäbrä-Iyäsus, Paris, Aresæ, 1993.

12. I. T ADDIA, L'Eritrea-colonia 1890-1952: paesaggi, strutture, uomini del colonialismo, Bologna, Franco Angeli, 1986. 